\title{
Laparoscopic splenectomy via the spleen bed in combination with selective esophagogastric devascularization for patients with cirrhotic portal hypertension: a single-institution experience
}

\author{
Xiaopei Hao, Kunfu Dai, Yuting He, Lianyuan Tao, Haibo Yu \\ Department of Hepatobiliary Surgery, People's Hospital of Zhengzhou University, Henan Provincial People’s Hospital, Zhengzhou, China
}

Videosurgery Miniinv 2020; 15 (3): 462-468

DOI: https://doi.org/10.5114/wiitm.2019.89655

\begin{abstract}
Introduction: The safety and feasibility of laparoscopic splenectomy plus selective esophagogastric devascularization (LSSD) via the spleen bed for cirrhotic portal hypertension have not been well studied.

Aim: To assess the safety and feasibility of LSSD via the spleen bed for patients with cirrhotic portal hypertension.

Material and methods: From June 2012 to December 2017, 423 patients suffering from portal hypertension and hypersplenism with liver cirrhosis underwent surgery in our department. One hundred and sixty-seven of these patients received totally LSSD, and the others received open splenectomy and esophagogastric devascularization (OSD). The characteristics, intraoperative and postoperative details and complications of the two groups were compared.

Results: The operations were successfully performed in all patients. Intraoperative blood loss volume and blood transfusion were similar between the two groups (all p-values > 0.05). Postoperative length of hospital stay and time to oral intake were significantly shorter, but operation time was longer in the LSSD group compared with the OSD group (all $p<0.05$ ). However, postoperative portal vein diameter was significantly smaller in the LSSD group ( $p<0.001)$. The postoperative grade of varices was significantly lower in the LSSD group $(p=0.030)$. No significant differences were detected between the two groups regarding postoperative liver function, but the incidences of pancreatic leakage, pleural effusion, and wound infections were higher in the OSD group (all $p<0.05)$.
\end{abstract}

Conclusions: LSSD via the spleen bed is safe and feasible for liver cirrhosis and portal hypertension.

Key words: liver cirrhosis, portal hypertension, laparoscopic splenectomy, selective esophagogastric devascularization.

\section{Introduction}

The severe complications of portal hypertension $(\mathrm{PH})$ caused by liver cirrhosis mainly include esophageal and gastric varices bleeding (EGVB) and hypersplenism [1]. Although nonsurgical treatment has improved, EGVB is still an unfavorable prognostic factor for patients with portal hypertension, with a 6-week mortality rate of approximately $20 \%$ [2]. Endoscopic therapy has been performed to control most cases of EGVB. However, the long-term outcomes of this approach are still unfavorable because new collateral vessels appear quickly, and severe thrombocytopenia due to hypersplenism significantly increases the risk of rebleeding by $60 \%$ [3]. 
Surgical intervention is currently the most reliable treatment option for cirrhosis. As an effective surgical procedure for EGVB and hypersplenism caused by PH, open splenectomy and esophagogastric devascularization (OSD) has been widely accepted by surgeons for a long time. However, the application of OSD has been limited due to the associated slow recovery, severe trauma, high incidence of postoperative complications and high morbidity. In 1998, Hashizume reported the feasibility of laparoscopic surgery for patients with sclerotherapy-resistant esophagogastric varices [4]. With the accumulation of experience and improvements to operating devices, a growing amount of evidence demonstrates that laparoscopy is superior to open surgery for managing $\mathrm{PH}[5,6]$.

To improve the curative effect and efficiency of the surgical procedure, laparoscopic surgeons have explored multiple technical modifications to splenectomy and devascularization [7-10]. However, limited studies have been published on the procedure for laparoscopic surgery.

\section{Aim}

The aim of the present study was to assess the safety and feasibility of the improved procedure for laparoscopic splenectomy plus selective esophagogastric devascularization (LSSD) via the spleen bed for patients with cirrhotic portal hypertension.

\section{Material and methods}

\section{Patients}

From June 2012 to December 2017, 423 consecutive patients who underwent LSSD and OSD for portal hypertension of cirrhotic origin were enrolled, and patients who had conversion to laparotomy were not included. All operations were completed by our surgical team. The surgical indications were based on the following criteria: (1) Child-Pugh class A and B; (2) portal hypertension resulting from liver cirrhosis, with a history of EGVB; (3) moderate to severe varices evaluated by preoperative esophagogastroscopy; (4) severe thrombocytopenia ( $\leq 50 \times 10^{9} /$ l) and/or leukopenia $\left(\leq 3 \times 10^{9} / \mathrm{l}\right)$ resulting from hypersplenism; and (5) no organic lesions in the heart, lung, kidney or other important organs. Supportive treatments were implemented to improve coagulation, anemia, liver function, portal pressure, and nutrition for all patients before the operation. Because this study is a retrospective study, written consent was not obtained from all participants, and the patients' data were analyzed anonymously. This study was approved by the Ethics Committee of Henan Provincial People's Hospital.

\section{Surgical techniques}

For LSSD, the laparoscopic procedure was performed with the patient in the right semi-decubitus position after general anesthesia. The port sites are shown in Photo 1. Port A: a 10-mm trocar was placed under or close to the umbilicus for the $30^{\circ}$ laparoscope, and a $\mathrm{CO}_{2}$ pneumoperitoneum was established through port $\mathrm{A}$; the intra-abdominal pressure was controlled at 12 to $14 \mathrm{~mm} \mathrm{Hg}(1 \mathrm{~mm} \mathrm{Hg}=$ $0.133 \mathrm{kPa}$ ). Two $5-\mathrm{mm}$ trocars were inserted under the xiphisternum and the left mid-abdomen, named ports $B$ and $C$, respectively. Port D: a $12-\mathrm{mm}$ trocar was inserted at the midpoint between the xiphisternum and navel. Port E: another 10-mm trocar was inserted at the midpoint between port $\mathrm{C}$ and the navel. The positions of the trocars were often corrected according to the length of the spleen.

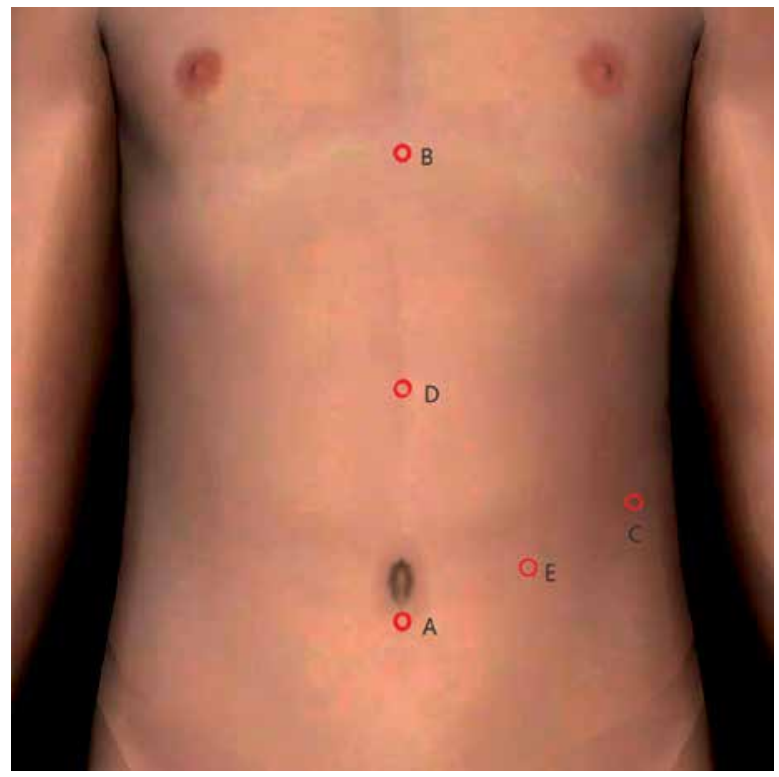

Photo 1. Positions of trocar ports in laparoscopic splenectomy and esophagogastric devascularization. A, E-10-mm trocar, B, C - 5-mm trocar, D-12-mm trocar 
The splenocolic ligament was routinely fully disconnected with the aid of a Harmonic Scalpel (Ethicon Endo-Surgery). The splenic artery was dissected via the dissociation of the gastropancreatic fold. If the splenic artery was at the superior margin of the pancreas, the artery was isolated from the vascular sheath and ligated easily with the aid of the Harmonic Scalpel and Hem-o-lok. If the splenic artery was behind the pancreas and hard to find, the spleen was directly dissected without isolating or ligating the splenic artery. The spleen was pushed to the medial side with a retractor, and the lower pole blood vessels were dissected by the Harmonic Scalpel and Ligasure Vessel Sealing System. Then, the lower side of the spleen was lifted by the surgeon with grasping forceps, and the connective tissue, including the splenorenal ligaments, was divided with the Harmonic Scalpel. In this process, the surgeon's grasping forceps could be braced against the top of the abdominal wall to stabilize the operative field. The upper side attachments, including the splenophrenic ligament, were dissected in a similar man-

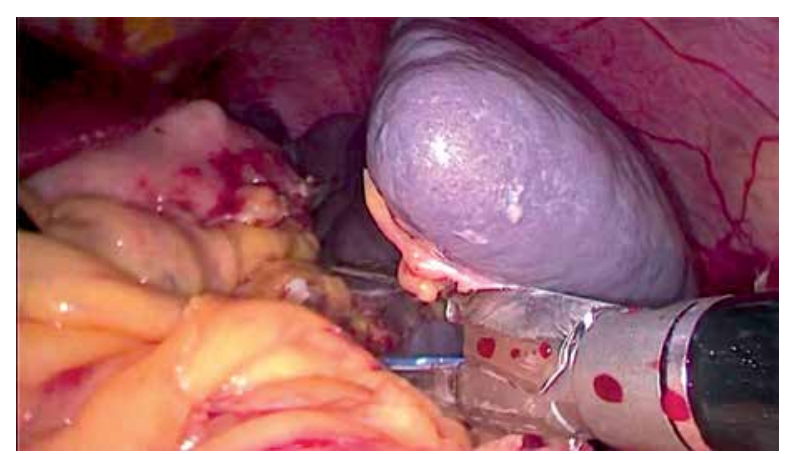

Photo 2. Echelon Flex 60 was also used to resect the spleen at the splenic hilum

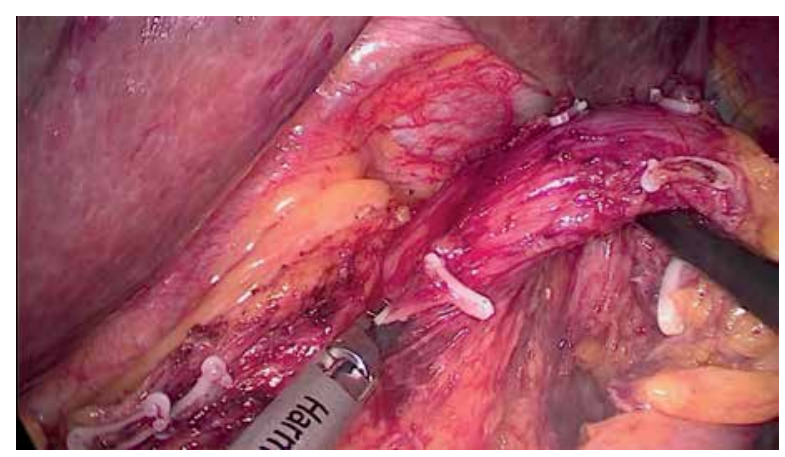

Photo 3. Laparoscopic esophagogastric devascularization with the Harmonic Scalpel and Hem-o-lock clamps ner. The splenic hilum was fully exposed by dividing the gastrosplenic ligament and the short gastric vessel. Normally, the short gastric vessels were well ligated with Hem-o-lok clamps. However, when the gastrosplenic ligament and the short gastric vessel were markedly dilated, an autosuture device (Linear Flex, Ethicon Endo-Surgery) may be a better choice for advancing the dissection. Then, the spleen was freed from all attachments and ligaments, except for the splenic hilum (Photo 2). Finally, the splenectomy was completed using an endoscopic linear stapler (Echelon Flex 60 Endopath) to transect the spleen at the splenic hilum. The spleen was cut into pieces, maneuvered into a retrieval sack and then removed through the enlarged E port (approximately $3 \mathrm{~cm}$ ). The partial E port was sutured, and the pneumoperitoneum was re-established. The spleen bed was carefully checked for bleeding, and any bleeding was completely stopped.

Selective devascularization began with the dissection of the left subphrenic vein and the posterior gastric vein. Then, the gastrohepatic ligament was disconnected, and the tunnel behind the lower esophagus was built. Subsequently, the branches of the distal gastric coronary vein, including the gastric and esophageal branches and superior esophageal branches, were devascularized with the aid of the Harmonic Scalpel and Hem-o-lock clamps. The stem of the gastric coronary vein was reserved close to the lesser curvature of the stomach. Finally, devascularization was accomplished (Photo 3); the perforating branches of the paraesophageal vein were completely free from 8 to $10 \mathrm{~cm}$ of the distal esophagus. OSD was performed as described previously [11].

\section{Clinical analysis}

A detailed clinical evaluation and biochemical investigation were performed for all enrolled patients. Platelet counts were routinely assessed. The grade of the esophageal varices was evaluated by esophagogastroscopy before and 3 months after the operation. Liver function was evaluated based on the Child-Pugh score. Portal vein thrombosis (PVT) was defined as thrombosis occurring in the portal venous system and diagnosed by computed tomography (CT) or ultrasonography. The length of the spleen, portal vein diameter and ascites were documented by CT or ultrasonography. Peritone- 
al fluid amylase was assayed daily for the first 3 postoperative days to monitor for postoperative pancreatic leakage. Operative mortality was defined as death within 30 days after the operation. The intraoperative and postoperative details and outcomes of the follow-up were retrospectively analyzed.

\section{Statistical analysis}

Continuous variables were expressed as the mean \pm standard deviation and compared using Student's $t$-test. Quantitative variables were compared using Fisher's exact test. $P<0.05$ was considered statistically significant. Statistical analyses were conducted using SPSS 19.0 software (SPSS Inc., Chicago, US).

\section{Results}

\section{Comparison of clinical characteristics of LSSD and OSD groups}

A comparison of the clinical characteristics between the two groups is presented in Table I. No significant differences were found between the two groups (all $p$-values $>0.05$ ).

\section{Operative details}

Both the laparoscopic procedure and open procedure were successfully performed in the two groups, and from the 171 patients initially selected in the LSSD group, 4 cases were excluded due to conversion. Regarding postoperative liver function, no significant differences were found in alanine transaminase (ALT), aspartate transaminase (AST) or total bilirubin (T-BIL) between patients in the two groups. In addition, the intraoperative blood loss and blood transfusion were similar between the two groups. The postoperative length of hospital stay and time to oral intake were much shorter in the LSSD group than in the OSD group, but the operation time was longer in the LSSD group than in the OSD group (all $p<0.001$ ). The postoperative portal vein diameter was much smaller in the LSSD group than in the OSD group $(p<0.001)$. Also, the grades of esophageal varices were improved in the two groups after the operation, and the severity of varices was lower in the LSSD group than in the OSD group $(p=0.030)$ (Table II).

\section{Postoperative complications}

As shown in Table III, no significant differences were detected in the rate of postoperative complications between the two groups, including postoperative upper gastrointestinal (GI) rebleeding and pulmonary infection, but the incidence of portal vein thrombosis, pancreatic leakage, pleural effusion, and wound infection was higher in the OSD group than in the LSSD group (all $p<0.05$ ). Two patients experienced upper GI rebleeding in the LSSD group: one with esophagogastric varices and the other with portal hypertension gastropathy. Three patients experienced upper GI rebleeding in the OSD group: 2 with esophagogastric varices and 1 with gastric ulcers. Patients who had these complications were all cured or experienced improvements after conservative medical treatment.

Table I. Characteristics of the patients in the two groups

\begin{tabular}{|c|c|c|c|}
\hline Variable & $\begin{array}{l}\text { LSSD group } \\
\quad(n=167)\end{array}$ & $\begin{array}{l}\text { OSD group } \\
(n=256)\end{array}$ & $P$-value \\
\hline Age [years] & $47.4 \pm 9.8$ & $48.6 \pm 12.2$ & 0.287 \\
\hline Sex: & & & 0.482 \\
\hline Male & 102 & 165 & \\
\hline Female & 65 & 91 & \\
\hline HBV infection: & & & 0.312 \\
\hline Yes & 136 & 218 & \\
\hline No & 31 & 38 & \\
\hline $\mathrm{ALT}[\mathrm{U} / \mathrm{I}]$ & $50.31 \pm 40.28$ & $47.29 \pm 36.99$ & 0.429 \\
\hline AST [U/I] & $40.96 \pm 27.06$ & $37.35 \pm 19.94$ & 0.116 \\
\hline T-BIL $[\mu \mathrm{mol} / \mathrm{l}]$ & $24.44 \pm 20.81$ & $22.17 \pm 16.76$ & 0.492 \\
\hline INR & $1.34 \pm 0.34$ & $1.32 \pm 0.31$ & 0.533 \\
\hline Child-Pugh classi & ication: & & 0.383 \\
\hline$A$ & 108 & 176 & \\
\hline B & 59 & 80 & \\
\hline Grade of varices: & & & 0.104 \\
\hline Moderate & 37 & 75 & \\
\hline Severe & 130 & 181 & \\
\hline $\begin{array}{l}\text { Portal vein } \\
\text { diameter }[\mathrm{mm}]\end{array}$ & $14.0 \pm 0.7$ & $14.1 \pm 0.6$ & 0.118 \\
\hline $\begin{array}{l}\text { Splenic length } \\
{[\mathrm{cm}]}\end{array}$ & $24.4 \pm 3.6$ & $23.9 \pm 3.5$ & 0.156 \\
\hline
\end{tabular}


Table II. Intraoperative and postoperative data of the two groups

\begin{tabular}{|lccc|}
\hline Variable & LSSD group $(n=167)$ & OSD group $(n=256)$ & $P$-value \\
\hline ALT $[\mathrm{U} / \mathrm{l}]$ & $57.22 \pm 47.28$ & $58.69 \pm 73.76$ & 0.863 \\
\hline AST $[\mathrm{U} / \mathrm{l}]$ & $71.84 \pm 65.87$ & $69.64 \pm 75.58$ & 0.841 \\
\hline T-BIL $[\mu \mathrm{mol} / \mathrm{I}]$ & $27.36 \pm 22.30$ & $22.86 \pm 12.82$ & 0.195 \\
\hline INR & $1.35 \pm 0.33$ & $1.34 \pm 0.25$ & 0.724 \\
\hline Operation time [min] & $272.3 \pm 96.2$ & $176.5 \pm 67.6$ & $<0.001$ \\
\hline Intraoperative blood loss [ml] & $510.6 \pm 424.30$ & $570.5 \pm 510.3$ & 0.209 \\
\hline Transfusion (RBC): & & & 0.417 \\
\hline Yes & 41 & 184 & $<0.001$ \\
\hline No & 126 & $14.8 \pm 5.7$ & $<0.001$ \\
\hline Postoperative length of hospital stay [days] & $11.5 \pm 4.6$ & $3.6 \pm 0.8$ & $<0.001$ \\
\hline Time to oral intake [days] & $3.2 \pm 0.8$ & $14.2 \pm 0.5$ & 0.030 \\
\hline Portal vein diameter [cm] & $14.0 \pm 0.6$ & & 201 \\
\hline Grade of varices: & & 55 & \\
\hline Mild to moderate & 145 & 22 & \\
\hline Severe & & & $<$ \\
\hline
\end{tabular}

Table III. Lists of postoperative complications in the LSSD and OSD groups

\begin{tabular}{|lccc|}
\hline Complication & LSSD group $(n=167)$ & OSD group $(n=256)$ & $P$-value \\
\hline Portal vein thrombosis $(n)$ & 28 & 77 & 0.002 \\
\hline Upper Gl rebleeding $(n)$ & 2 & 3 & 0.981 \\
\hline Pancreatic leakage $(n)$ & 1 & 16 & 0.004 \\
\hline Pleural effusion $(n)$ & 16 & 64 & $<0.001$ \\
\hline Pulmonary infection $(n)$ & 2 & 10 & 0.101 \\
\hline Wound infection $(n)$ & 0 & 7 & 0.031 \\
\hline Gl - gastrointestinal. & & &
\end{tabular}

\section{Discussion}

As a crucial part of LSSD, laparoscopic splenectomy (LS) largely determines the completion rate of laparoscopic surgery, and a slight mistake may cause massive hemorrhage and confers a high risk of conversion to open surgery [12]. With the exploration of minimally invasive treatment for $\mathrm{PH}$ and significant advances in laparoscopic techniques, we established a new approach for LS. This improved procedure not only modularizes the splenectomy procedure to optimize the surgical process but also provides enough operating space for the subsequent selective devascularization procedure.

Here, we share the following experience for performing LS: (1) First, the initial separation of the splenic artery is suggested since this separation can avoid massive hemorrhage during surgery and shrink the enlarged spleen through blood recirculation. If it is difficult to find the splenic artery, separation is not required, since the separation may cause pancreatic injury or even lead to pancreatic leakage. (2) Second, for vessels (both arteries and veins) less than $3 \mathrm{~mm}$ in diameter, ultrasound scalpels can be safely 
and feasibly used to facilitate dissection, which ensures a clear operative field and a more thorough disconnection. (3) Third, the surgical approach of "tunnel-building" combined with an endoscopic linear stapler effectively avoids the blood vessels of the splenic hilum, which lowers the risk of bleeding.

Bleeding is the most critical complication during LSSD $[13,14]$. In the present study, we demonstrated that the volume of intraoperative blood loss and the number of cases requiring blood transfusion were the same between the LSSD and the OSD groups, which indicates that LSSD can effectively control bleeding to the same degree as OSD. This result can be attributed to the meticulous dissection and the application of advanced laparoscopic instruments, such as the Harmonic Scalpel, Ligasure and Hem-olock clamp.

Although the operation time was longer for the LSSD group than for the OSD group, the liver function was not significantly different between the two groups, and we speculate that LSSD has potential advantages in protecting portal vein perfusion. We believe that as experience in laparoscopic surgery accumulates, the operation time will be gradually shortened.

PVT is a poten-tially fatal postoperative complication of both LSSD and OSD. The incidence of PVT was much higher after LSSD than after OSD [11], but the mechanism is poorly understood. However, our results show that the rate of PVT was significantly lower in the LSSD group than in the OSD group, which we speculate may be due to two potential reasons: first, the selective devascularization procedure can retain a spontaneous shunt for the body, which is helpful for reducing the portal vein pressure; second, the portal vein diameter of the LSSD group was significantly smaller than that of the OSD group, indicating that the portal vein pressure was relieved to some extent. A reduced portal vein pressure significantly improves postoperative hemodynamics and relieves the damage of the venous intima, which leads to a reduction in platelet adhesion and aggregation.

latrogenic pancreatic injury is another common surgical complication after splenectomy and results in poor or even fatal outcomes in some patients [15]. The incidence of pancreatic injury is nearly $16 \%$ in patients who undergo open splenectomy but is much lower in patients who undergo LS (only 1-2\%) [16]. The rate of pancreatic leakage in the LSSD group was effectively decreased compared with that in the OSD group because the modified approach offers better visualization and operation space around the splenic hilum and facilitates the transection of the splenic hilum away from the pancreatic tail. In addition, the use of an endoscopic linear stapler is another important reason for the reduced incidence of pancreatic injury and leakage.

Our study also demonstrated that the use of LSSD provides the following advantages over OSD: earlier bowel activity and oral intake, shorter hospital stay, lower postoperative severity of varicose veins, less trauma, less postoperative pain, and lower rate of pleural effusion; these advantages are in accordance with other reports [6]. Our original intention was to compare the efficacy of totally laparoscopic surgery and open surgery, and considering the fact that only 4 patients had conversion (which was mainly due to extensive perisplenic adhesions rather than bleeding), so patients who had conversion were excluded to avoid potential factors affecting the results of our study. However, our results may be limited by the fact that all patients were from a single center, and bias may be generated due to the short follow-up duration. Multicenter studies and extended follow-up periods are needed to further validate the feasibility of this surgical procedure. In conclusion, LSSD via the spleen bed is a safe and feasible procedure for experienced laparoscopic surgeons to manage patients with portal hypertension.

\section{Conclusions}

The LSSD via the spleen bed is safe and feasible for liver cirrhosis and portal hypertension.

\section{Conflict of interest}

The authors declare no conflict of interest.

\section{References}

1. Lin J, Liu Q, Liang Z, et al. Laparoscopic selective esophagogastric devascularization and splenectomy for patients with cirrhotic portal hypertension. Videosurgery Miniinv 2019; 14: 187-94.

2. Luigiano C, Iabichino G, Judica A, et al. Role of endoscopy in management of gastrointestinal complications of portal hypertension. World J Gastrointest Endosc 2015; 7: 1-12.

3. Bari K, Garciatsao G. Treatment of portal hypertension. World J Gastroenterol 2012; 18: 1166-75.

4. Hashizume M, Tanoue K, Morita M, et al. Laparoscopic gastric devascularization and splenectomy for sclerotherapy-resistant 
esophagogastric varices with hypersplenism. J Am Coll Surg 1998; 187: 263-70.

5. Hong D, Cheng J, Wang Z, et al. Comparison of two laparoscopic splenectomy plus pericardial devascularization techniques for management of portal hypertension and hypersplenism. Surg Endosc 2015; 29: 3819-26.

6. Chen H, Yang F, Li TT, et al. Comparison of efficacy of laparoscopic and open splenectomy combined with selective and nonselective pericardial devascularization in portal hypertension patients. Surg Laparosc Endosc Percutan Tech 2018; 28: 401-3.

7. Ji B, Wang Y, Zhang P, et al. Anterior versus posterolateral approach for total laparoscopic splenectomy: a comparative study. Int J Med Sci 2013; 10: 222-9.

8. Xu J, Zhao L, Wang Z, et al. Single-incision laparoscopic splenectomy for massive splenomegaly combining gastroesophageal devascularization using conventional instruments. Surg Laparosc Endosc Percutan Tech 2014; 24: e183.

9. Podevin G, Victor A, De Napoli S, et al. Laparoscopic splenectomy: comparison between anterior and lateral approaches. J Laparoendosc Adv Surg Tech A 2011; 21: 865-8.

10. Tian G, Li D, Yu H, et al. Laparoscopic splenectomy: posterolateral approach in patients with liver cirrhosis and portal hypertension with platelet count lower than $1 \times 109 /$. Videosurgery Miniinv 2018; 13: 454-9.

11. Zhe C, Jian-wei L, Jian C, et al. Laparoscopic versus open splenectomy and esophagogastric devascularization for bleeding varices or severe hypersplenism: a comparative study. J Gastrointest Surg 2013; 17: 654-9.

12. Bani Hani MN, Qasaimeh GR, Bani-Hani KE, et al. Laparoscopic splenectomy: consensus and debatable points. S Afr J Surg 2010; 48: 81-4.

13. Schepis F, Turco L, Bianchini M, et al. Prevention and management of bleeding risk related to invasive procedures in cirrhosis. Semin Liver Dis 2018; 38: 215-29.

14. Kockerling D, Nathwani R, Forlano R, et al. Current and future pharmacological therapies for managing cirrhosis and its complications. World J Gastroenterol 2019; 25: 888-908.

15. Tsutsumi N, Tomikawa M, Akahoshi T, et al. Pancreatic fistula after laparoscopic splenectomy in patients with hypersplenism due to liver cirrhosis: effect of fibrin glue and polyglycolic acid felt on prophylaxis of postoperative complications. Am J Surg 2016; 212: 882-8

16. Vecchio R, Gelardi V, Intagliata E, et al. How to prevent intraoperative risks and complications in laparoscopic splenectomy. G Chir 2010; 31: 55-61.

Received: 9.09.2019, accepted: 18.10.2019. 